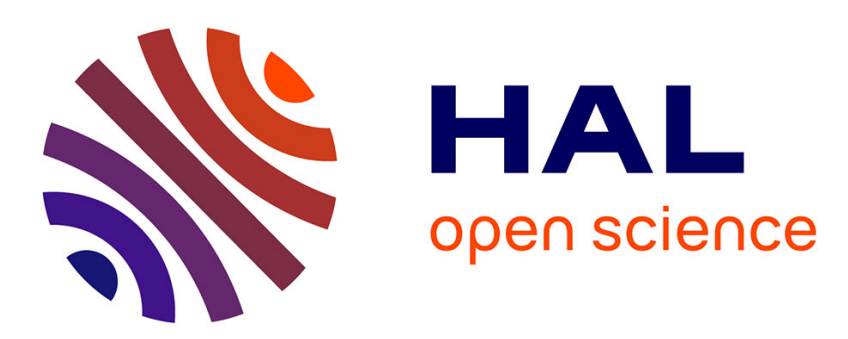

\title{
Fluid and kinetic modelling for non-local heat transport in magnetic fusion devices
}

Guido Ciraolo, Hugo Bufferand, Pierfrancesco Di Cintio, Philippe Ghendrih, Stefano Lepri, Roberto Livi, Yannick Marandet, Eric Serre, Patrick Tamain, Matteo Valentinuzzi

\section{To cite this version:}

Guido Ciraolo, Hugo Bufferand, Pierfrancesco Di Cintio, Philippe Ghendrih, Stefano Lepri, et al.. Fluid and kinetic modelling for non-local heat transport in magnetic fusion devices. Contributions to Plasma Physics, 2018, 58 (6-8), pp.457-464. 10.1002/ctpp.201700222 . hal-02113953

\section{HAL Id: hal-02113953 \\ https://hal-amu.archives-ouvertes.fr/hal-02113953}

Submitted on 29 Apr 2019

HAL is a multi-disciplinary open access archive for the deposit and dissemination of scientific research documents, whether they are published or not. The documents may come from teaching and research institutions in France or abroad, or from public or private research centers.
L'archive ouverte pluridisciplinaire HAL, est destinée au dépôt et à la diffusion de documents scientifiques de niveau recherche, publiés ou non, émanant des établissements d'enseignement et de recherche français ou étrangers, des laboratoires publics ou privés. 


\title{
Fluid and kinetic modelling for non-local heat transport in magnetic fusion devices
}

\author{
Guido Ciraolo $^{\mathbf{1}^{*}} \mid$ Hugo Bufferand ${ }^{\mathbf{1}} \mid$ Pierfrancesco Di Cintio $^{2,3}$ | Philippe Ghendrih $^{\mathbf{1}} \mid$ \\ Stefano Lepri ${ }^{3,4}$ | Roberto Livi ${ }^{3,4,5}$ | Yannick Marandet ${ }^{6}$ | Eric Serre $^{7}$ \\ Patrick Tamain $^{1}$ | Matteo Valentinuzzi ${ }^{1}$
}

${ }^{1}$ IRFM, CEA, St Paul Lez Durance, France

${ }^{2}$ IFAC, CNR, Sesto Fiorentino, Italy

${ }^{3}$ INFN, Sezione Firenze, Sesto Fiorentino, Italy

${ }^{4}$ ISC, CNR, Sesto Fiorentino, Italy

${ }^{5}$ Dipartimento di Fisica e Astronomia and CSDC,

Universitá di Firenze, Sesto Fiorentino, Italy

${ }^{6}$ PIIM, CNRS - Aix-Marseille Université,

Marseille, France

${ }^{7}$ M2P2, CNRS - Aix-Marseille Université,

Marseille, France

*Correspondence

Guido Ciraolo, CEA IRFM, 13108 St Paul Lez

Durance, France.

Email: guido.ciraolo@cea.fr

Funding Information

This research was supported by the $\mathrm{H} 2020$

Euratom, grant agreement No 633053 for the

project WP17-ENR.
In order to improve the presently used ad hoc flux limiter treatment of parallel heat flux transport in edge plasma fluid codes, here, we consider a generalized version of the Fourier law implementing a non-local kernel for the heat flux computation. The Bohm boundary condition at the wall is recovered, introducing a volumetric loss term representing the contribution of suprathermal particles to the energy out flux. As expected, this contribution is negligible in the strongly collisional regime, while it becomes more and more dominant for marginally and low-collisional regimes. In the second part of the paper, we consider a kinetic approach where collisions are considered using the multi-particle collision algorithm. Kinetic simulation results at medium and low collisionality are also reported.

KEYWORDS

Coulomb collisions, fluid models, heat transport, kinetic equations

\section{1 | INTRODUCTION}

Modelling parallel heat transport in edge tokamak plasma is a crucial issue for predictions of power loads on divertor targets. In the operational regimes of interest for a magnetic fusion device, a significant temperature gradient will build up along the field line between the upstream hot region that acts as a heat source and the colder plasma region at the wall that acts as a sink. Numerical estimations of edge and scrape-off layer (SOL) plasma rely mainly on two-dimensional (2D) transport codes like, for example, SOLEDGE2D, ${ }^{[1]}$ SOLPS-ITER, ${ }^{[2]}$ EDGE2D, ${ }^{[3]}$ SONIC, ${ }^{[4]}$ and UEDGE. ${ }^{[5]}$ These numerical tools are based on a fluid approach and a collisional closure with the so-called Spitzer-Härm (hereafter SH, see Ref. 6) expression for the parallel heat flux

$$
q_{\|}(x)=-\kappa(x) \nabla_{\|} T(x),
$$

where the thermal conductivity $\kappa(x)$ is computed in the strong collisionality assumption (i.e., considering a small departure from the Maxwellian distribution function) and reads

$$
\kappa(x)=\kappa_{0} T(x)^{5 / 2}
$$

When collisionality drops, the classical Fourier law fails in describing heat transport, and the expression above leads to overestimated heat fluxes (see, e.g., Refs. 7, 8 and references therein). 
FIGURE 1 Radial profiles of flux surface-averaged transport coefficients used in SOLEDGE2D-EIRENE simulations inspired by Chankin et al. ${ }^{[10]}$

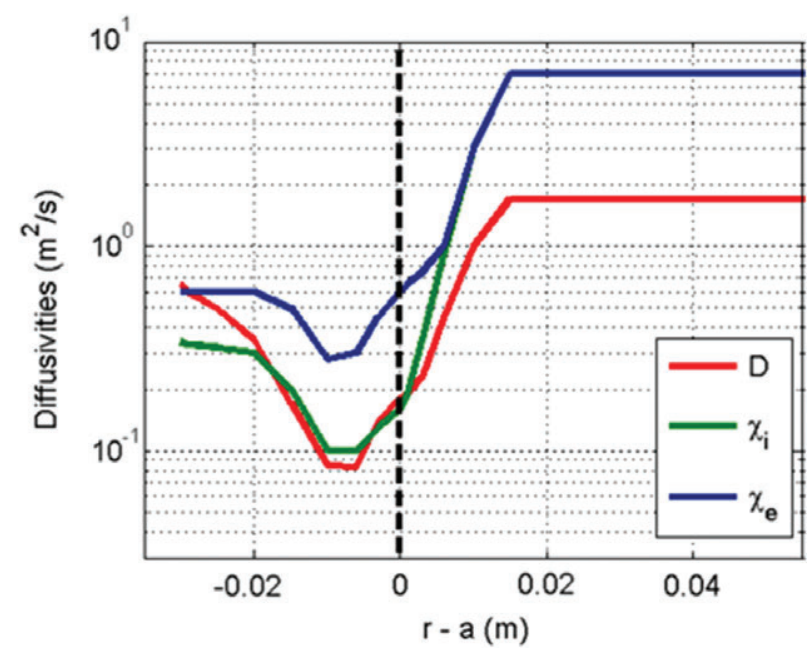

Typically, in order to avoid unphysical divergences in the SH expression for the heat flux, an ad hoc flux limiter (FL) correction is introduced with the following harmonic average between the free streaming heat flux $q_{F S}=n v_{t h} T$ and the collisional expression $q_{S H}$ :

$$
q_{\|}=\left(\frac{1}{q_{S H}}+\frac{1}{\alpha n v_{\mathrm{th}} T}\right)^{-1} .
$$

In the formulae above, $v_{t h}$ is the thermal velocity, $n$ the plasma density, and $\alpha$ is a free parameter ranging from 0.1 to 3 characteristic values.

In Figure 2, we report an example of the strong impact that such a FL expression can have on the predictions obtained from transport codes on energy fluxes at the wall. We consider a SOLEDGE2D simulation for WEST configuration ${ }^{[9]}$ in pure deuterium with an input power of $P_{i n}=4 \mathrm{MW}$ and a gas puff activated in the private flux region with an injection rate equal to $4 \times 10^{21}$ atoms per second. The radial transport coefficients $D$ for density, $\chi_{i}$ for ion temperature, and $\chi_{e}$ for electron temperature are reported in Figure 1. They are settled equal to the ones presented by Ciraolo et al. ${ }^{[11]}$ and, waiting for measurements on WEST plasmas, have been chosen taking into account parameters that have been adjusted to match experimental mid-plane profiles of a H-mode ASDEX Upgrade plasma (see Ref. 10).

The SOLEDGE-EIRENE simulations are performed considering three different expressions for the electron heat flux transport (while the ion heat flux is always computed using the FL expression with $\alpha=0.2$ ): in the first simulation, we do not activate the FL, and the electron heat flux is computed using the SH expression. In the second and third simulations, the electron heat flux is computed using the FL expression given by Equation 3, with $\alpha=0.3$ and $\alpha=0.15$, respectively. In Figure 2 (left panel), we demonstrate with a 2D colour map the electron temperature in a WEST poloidal section obtained in the SH case. In the right panel, the comparison between the electron temperature profiles at the outer strike point obtained from these three different cases (SH, FL with $\alpha=0.3$, and FL with $\alpha=0.15$ ) is presented. We note that there is a strong reduction of the temperature peak value between the SH case, and the one computed with an FL equals to $\alpha=0.15$.

In order to improve the presently used ad hoc FL treatment of parallel heat flux transport in edge plasma codes, we consider a fluid description with the generalized version of the Fourier law implementing a non-local kernel for the heat flux computation as proposed, for example, in the paper by Luciani and co-authors. ${ }^{[12]}$

$$
q_{N L}(x)=-\int w\left(x, x^{\prime}\right) \kappa\left(x^{\prime}\right) \nabla T\left(x^{\prime}\right) d x^{\prime},
$$

where $q_{N L}$ is the non-local heat flux, $w$ the delocalization kernel, $\kappa$ the classical SH collisional conductivity, and $T$ the temperature. The simplest phenomenological form of the kernel is the one in which memory decays exponentially in space,

$$
w\left(x, x^{\prime}\right)=\frac{1}{2 \lambda\left(x^{\prime}\right)} \exp \left(-\left|x-x^{\prime}\right| / \lambda\left(x^{\prime}\right)\right),
$$

where $\lambda\left(x^{\prime}\right)$ is the local electron mean free path at the position $x^{\prime}$.

We have shown in Ref. 13 that solving the equation $\partial_{x} q_{N L}(x)=S$ using this non-local expression for the heat flux computation can lead to discontinuities in the temperature profile if the source term $S$ is very localized in space, such as, for example, in the case of the interaction with the wall, and the collisionality takes medium and low values as it can happen in the SOL plasma of tokamaks. In order to overcome this issue, we have introduced (see again Ref. 13) the following expression for the heat flux:

$$
q_{N L, T}(x)=q_{N \tilde{L}}(x)+q_{B C, 0} \exp \left(-\frac{x}{\lambda}\right)+q_{B C, L_{\|}} \exp \left(\frac{x-L_{\|}}{\lambda}\right) .
$$



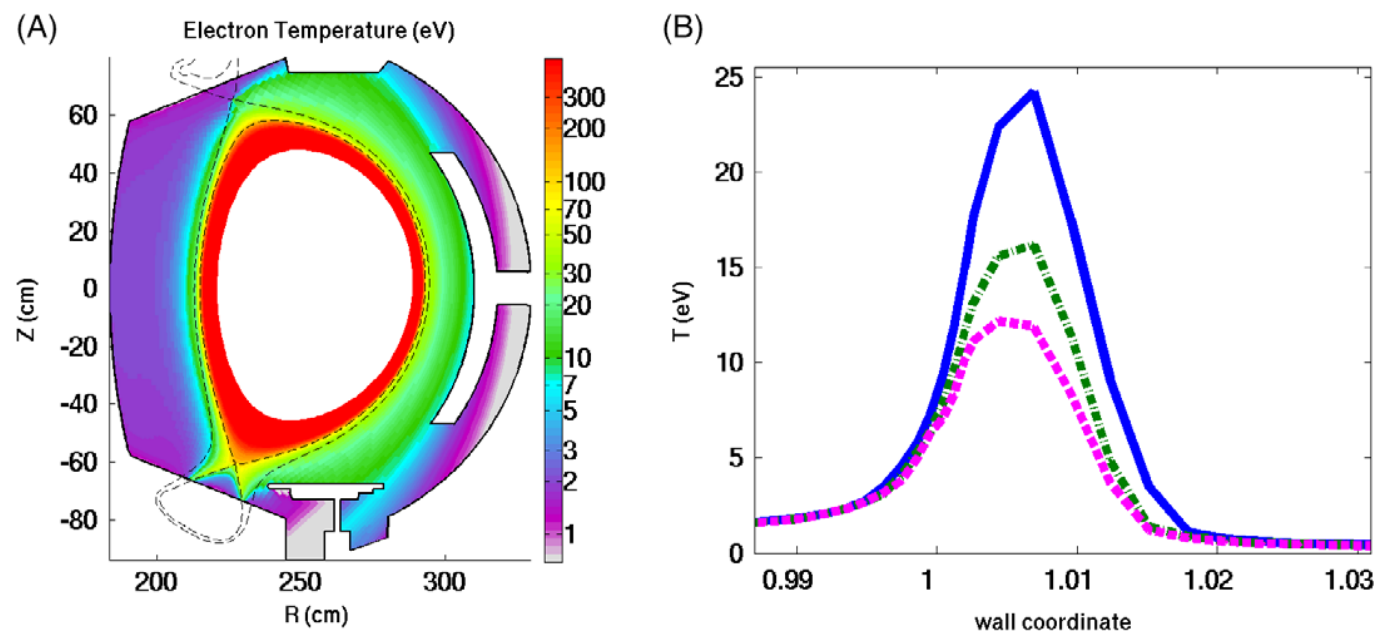

FIGURE 2 Left Panel: contour plot of the electron temperature in the poloidal section obtained from a SOLEDGE2D-EIRENE simulation (using the Spitzer-Härm [SH] expression for the electron heat flux computation) with input power $P_{i n}=4 \mathrm{MW}$ and a gas puff of $4 \times 10^{21}$ atoms per second activated in the private flux region. Right panel: electron temperature profiles on the outer divertor target computed using SH expression (solid blue line), flux limiter expressions with parameter $\alpha=0.3$ (dash-dot green line), and $\alpha=0.15$ (dashed pink line)

This expression exhibits a first term describing the non-local heat flux computed from the continuous temperature gradient expression in the plasma. The two last terms represent the impact of the boundary condition in the heat flux and the effect that decays exponentially away from the wall. They describe the long range influence of the boundary conditions. The values $q_{b c, 0}$ and $q_{b c, L_{\|}}$are adjusted to match the sheath boundary condition for the heat flux, namely, $q_{s e}=\gamma n_{\text {wall }} c_{s} T_{\text {wall }}$ at both ends, where $\gamma$ is the so-called sheath transmission coefficient. We note that the value of the sheath heat transmission coefficient gamma depends also on the collisionality of the system and can take very large values when the high-energy tail exists (see, e.g., Refs. 14, 15). However, for steady-state conditions like the one considered in this paper, the sheath transmission coefficients are quite constant for a large range of collisionality values.

\section{2 | NON-LOCAL HEAT TRANSFER IN FLUID MODELS: APPLICATION TO 1D SOL WITH LOCALIZED PARTICLE AND ENERGY SOURCES}

We consider a one-dimensional (1D) model of SOL plasma where we solve the standard equations for density, parallel momentum, and ion and electron energy balance with standard Bohm boundary conditions, including the non-local expression for heat flux introduced above. Localized sources of density (particle recycling) and energy (e.g., Radio Frequency (RF) heating for both electrons and ions) have been added as follows. For the particle source, simulating a recycling source term we have imposed

$$
S_{n}(x)=S_{n}^{0}\left[\exp \left(-\frac{x}{0.1 L_{\|}}\right)+\exp \left(-\frac{L_{\|}-x}{0.1 L_{\|}}\right)+0.005\right],
$$

while for the energy sources, we have used Gaussian-shaped sources located in the middle of the field line. The width of the energy source is controlled by $\lambda_{E}$ and reads

$$
S_{E e, i}=S_{E e, i}^{0} \exp \left(-\left(\frac{x}{\lambda_{E}}-\frac{L_{\|}}{2 \lambda_{E}}\right)^{2}\right) .
$$

Here, we report two cases obtained by varying the amplitude of the energy source and producing a first case at medium collisionality $v^{\star}=60$ and a second one at low collisionality with $v^{\star}=4$, where $v^{\star}=L_{\|} / \lambda$ with $\lambda$ the electron mean free path. We note that, for the medium collisionality case (see Figure 3 ) the non-local expression collapses onto the standard SH expression, with a very small contribution coming from the non-local terms related to the influence of the boundary conditions. However, the $q_{e, B C}$ contribution is, as expected, non-negligible very close to the wall; for reference, see the dotted line in Figure 3. On the contrary, when the collisionality drops, the non-local expression is able to take into account the influence of the boundary conditions on the whole domain. In Figure 4, it appears clear that the contribution from the $q_{e, B C}$ expression to the total parallel heat flux is non-negligible on the entire domain and of the same order of magnitude of the conductive part. Interestingly, thanks to the proposed non-local expression, we can also recover the shape of the energy source into the temperature profile, which is Gaussian in the energy source region (see Figure 4, left panel).

In next section, we introduce the kinetic modelling of heat transfer, which will be used for a first analysis of the results obtained in this section. 
FIGURE 3 Left panel: electron temperature profile along $\times$ parallel to magnetic field $B$ from upstream position $(x=39 \mathrm{~m})$ to the wall $(x=78 \mathrm{~m})$ obtained from SOLEDGE1D simulation at $v^{\star}=60$. The solid line represents the results obtained considering the non-local heat flux expression, the dashed line using the Spitzer-Härm expression, and the dashed-dotted line the flux limiter expression with the free parameter $\alpha=0.15$. Right panel: heat flux profile along $\times$ parallel to $B$ from upstream position to the wall. The solid blue line is the sum of the contribution from the electron conductive term (reported with dashed line and dominant in this case) and the contribution from the $q_{e, B C}$ expression (see Equation 6) represented in dotted line, representing the long-range influence of the boundary conditions. The red solid line is the contribution from the remaining extra terms (e.g. convective, ions), while solid black line is the total heat flux
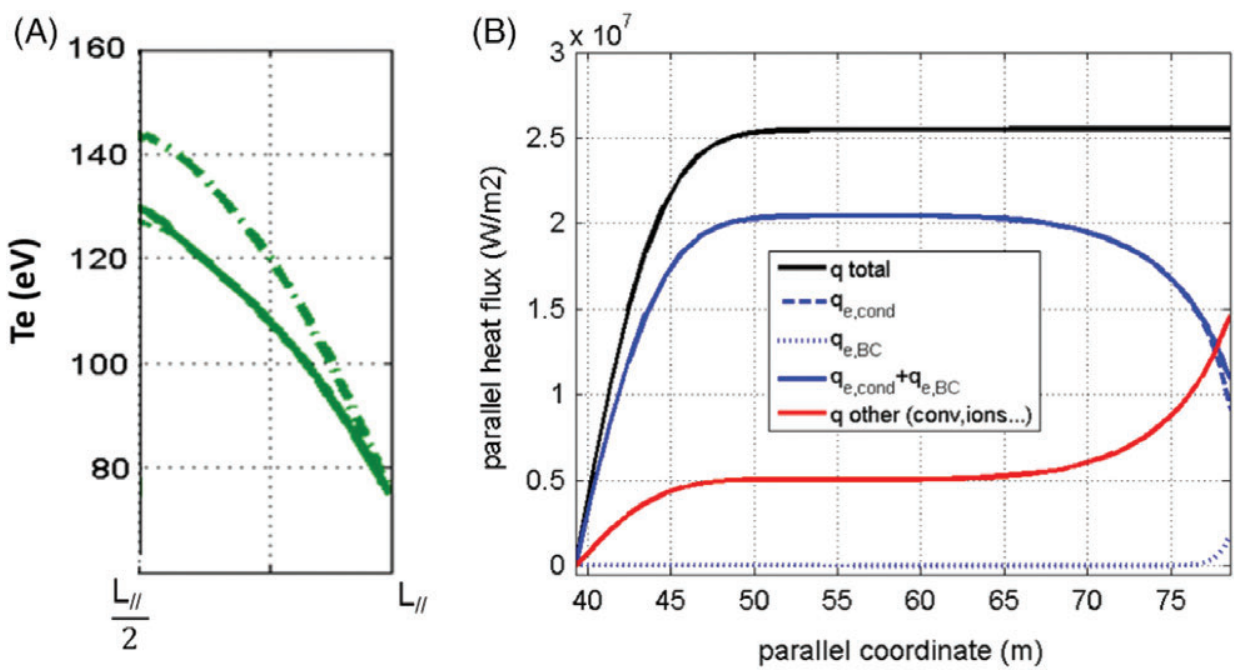

FIGURE 4 Left panel: electron temperature profile along $\times$ parallel to magnetic field $B$ from upstream position $(x=39 \mathrm{~m})$ to the wall $(x=78 \mathrm{~m})$ obtained from SOLEDGE1D simulation at $v^{\star}=4$. Line types in figures are the same as those in Figure 3. Right panel: heat flux profile along $\times$ parallel to $B$ from upstream position to the wall. Line types in figures are the same as those in Figure 3. In this case, the $q_{e, B C}$ contribution (dotted blue line) related to non-local effects is very large
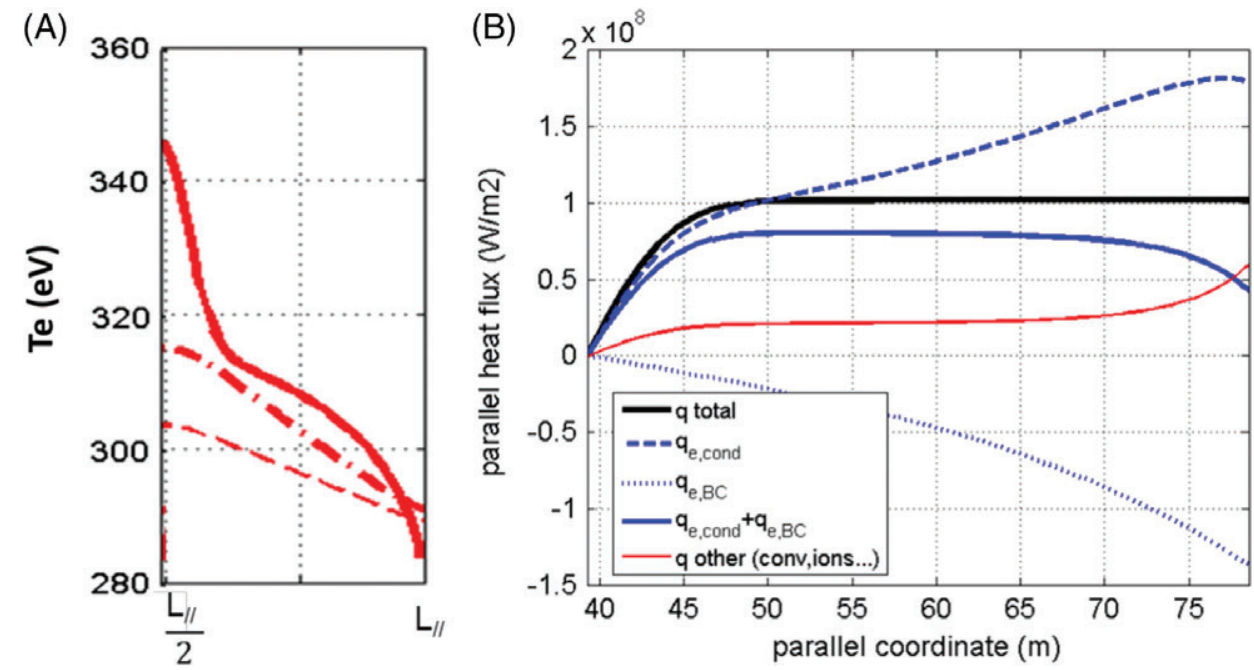

\section{3 | KINETIC MODELLING OF HEAT TRANSFER}

From the kinetic point of view, weakly collisional plasmas are usually studied in terms of their phase-space distribution function $f(\mathbf{r}, \mathbf{v})$ by means of the so-called Vlasov-Fokker-Planck equation (see, e.g., Refs. 16-18) that reads for the electron components as

$$
\frac{\partial f}{\partial t}+\mathbf{v} \cdot \nabla_{\mathbf{r}} f-\frac{e}{m_{e}}\left(\mathbf{E}+\frac{\mathbf{v}}{c} \times \mathbf{B}\right) \cdot \nabla_{\mathbf{v}} f=\nabla_{\mathbf{v}} \cdot\left(\nu \nabla_{\mathbf{v}} f\right) .
$$

In the equation above, the diffusion coefficient $v$ appearing in the velocity-space diffusive term at the right-hand side could be in principle an explicit function of velocity or be dependent on position through the local number density $n(\mathbf{r})=\int f d \mathbf{v}$, see Refs. 19, 20.

Equation 9 can be easily integrated with standard implicit Eulerian codes in the $1 \mathrm{D} 1 \mathrm{~V}$ and $1 \mathrm{D} 2 \mathrm{~V}$ cases, ${ }^{[17]}$ adopting standard Max well solvers to account for the self-consistent electric and magnetic fields $\mathbf{E}$ and $\mathbf{B}$. Already in two spatial dimensions (2D2V or 2D3V), such an approach rapidly becomes numerically expensive, and we therefore rely on particle* based (semi-)Lagrangian methods such as particle-in-cell (PIC; see, e.g., Ref. 21). Including the contribution of collisions in cell-based PIC codes is usually time consuming and model dependent; here, we used a stochastic approach based on the multi-particle collision (hereafter MPC) technique. 


\section{1 | Multi-particle collision method}

Originally introduced by Malevanets and Kapral ${ }^{[22]}$ for the simulation of complex fluids (e.g., polymers in solution, colloidal fluids), in three spatial dimensions, the MPC scheme partitions the system of $N_{p}$ particles in $N_{c}$ cells. ${ }^{\dagger}$ Between two standard propagation steps, inside each cell, the particle velocities in the cell's centre of mass $\delta \mathbf{v}_{j}=\mathbf{v}_{j}-\mathbf{u}_{i}$ are rotated of an angle $\varphi$ around a random axis $\mathbf{R}$ and then converted back to the simulation frame so that for the $j$-th particle in cell $i$

$$
\mathbf{v}_{j}^{\prime}=\mathbf{u}_{i}+\delta \mathbf{v}_{j, \perp} \cos (\varphi)+\left(\delta \mathbf{v}_{j, \perp} \times \mathbf{R}\right) \sin (\varphi)+\delta \mathbf{v}_{j, \|},
$$

where $\delta \mathbf{v}_{j, \perp}$ and $\delta \mathbf{v}_{j, \|}$ are the relative velocity components perpendicular and parallel to $\mathbf{R}$, respectively. Such an operation exactly conserves in each cell the total kinetic energy $K_{i}$ and the three components of the momentum $\mathbf{P}_{i}$. For an extensive proof of the conservation laws, see Appendix A in Ref. ${ }^{[23]}$. In addition, it is also possible to conserve the component of the angular momentum $\mathbf{L}$ parallel to $\mathbf{R}$ by choosing $\varphi$ so that

$$
\sin (\varphi)=-\frac{2 a_{i} b_{i}}{a_{i}^{2}+b_{i}^{2}} ; \quad \cos (\varphi)=\frac{a_{i}^{2}-b_{i}^{2}}{a_{i}^{2}+b_{i}^{2}},
$$

with cell-dependent coefficients $a_{i}$ and $b_{i}$ given by

$$
a_{i}=\left.\sum_{j=1}^{N_{i}}\left[\mathbf{r}_{j} \times\left(\mathbf{v}_{j}-\mathbf{u}_{i}\right)\right]\right|_{z} ; \quad b_{i}=\sum_{j=1}^{N_{i}} \mathbf{r}_{j} \cdot\left(\mathbf{v}_{j}-\mathbf{u}_{i}\right) .
$$

In the formulae above, $\mathbf{r}_{j}$ are the particles position vectors, and the notation $\mathrm{I}_{z}$ means that one is taking (without loss of generality) the component of the vector $\mathbf{A}_{i}$ parallel to the $z$ axis of the simulation's coordinate system. For 2D systems, Equation 10 reduces to $\mathbf{v}_{j}^{\prime}=\mathbf{u}_{i}+\mathbf{G}_{\varphi, i} \cdot \delta \mathbf{v}_{j}$, where $\mathbf{G}_{\varphi, i}$ is a 2 D rotation matrix of an angle $\varphi$ chosen according to relations; ${ }^{[10,13]}$ see Ref. 23. In both $2 \mathrm{D}$ and $3 \mathrm{D}$ cases, the generalization to multi-mass models is straightforward and implies the substitution of velocity vectors with momentum vectors.

In one dimension, the multi-particle collision instead involves a velocity sign inversion with a momentum shift (see also Ref. 24), and the two conserved quantities are the linear momentum $P_{i}$ and the kinetic energy $K_{i}$. During the collision step, the stochastic momentum shifts $w_{j}$ are extracted for each particle from a normal distribution depending on the cell temperature so that the conservation of $P_{i}$ and $K_{i}$ now reads

$$
\begin{aligned}
& P_{i}=\sum_{j=1}^{N_{i}} m_{j} v_{j}=\sum_{j=1}^{N_{i}} m_{j} v_{j}^{\prime}=\sum_{j=1}^{N_{i}}\left(c_{i} w_{j}+d_{i} m_{j}\right) \\
& K_{i}=\frac{1}{2} \sum_{j=1}^{N_{i}} m_{j} v_{j}^{2}=\frac{1}{2} \sum_{j=1}^{N_{i}} m_{j} v_{j}^{\prime 2}=\frac{1}{2} \sum_{j=1}^{N_{i}} m_{j}\left(c_{i} w_{j} / m_{j}+d_{i}\right)^{2},
\end{aligned}
$$

where $N_{i}$ is the number of particles in cell $i ; m_{j}$ and $v_{j}$ are the $j$-th particles mass and velocity, respectively; and $c_{i}$ and $d_{i}$ are unknown cell-dependent quantities, respectively. Equations 13 constitute a linear system that has to be solved for $c_{i}$ and $d_{i}$. We define the stochastic momentum and kinetic energy increments as

$$
P_{i}^{*}=\sum_{j=1}^{N_{i}} w_{j} ; \quad K_{i}^{*}=\frac{1}{2} \sum_{j=1}^{N_{i}} w_{j}^{2} / m_{j},
$$

and rescale them, together with $P_{i}$ and $E_{i}$, by the total mass in cell $i, M_{i}=\sum_{j=1}^{N_{i}} m_{j}$ as $\tilde{P}_{i}^{*}=P_{i}^{*} / M_{i}, \tilde{P}_{i}=P_{i} / M_{i}, \tilde{K}_{i}^{*}=K_{i}^{*} / M_{i}$, and $\tilde{K}_{i}=K_{i} / M_{i}$. The coefficients $c_{i}$ and $d_{i}$ are then easily computed as

$$
c_{i}=\sqrt{\frac{2 \tilde{K}_{i}-\tilde{P}_{i}^{2}}{2 \tilde{K}_{i}^{*}-\tilde{P}_{i}^{* 2}}} ; \quad d_{i}=\tilde{P}_{i}-\tilde{P}_{i}^{*} c_{i},
$$

so that the new velocities after the multi-particle collision finally read $v_{j}^{\prime}=c_{i} w_{j} / m_{j}+d_{i}$.

In a series of papers on the anomalous diffusion and heat transfer in $1 \mathrm{D}$ one-component plasmas, ${ }^{[23-26]}$ we have applied a hybrid PIC-MPC technique where velocity exchange inside the cells is conditioned to an interaction probability $\mathcal{P}_{i}$ dependent on the local plasma parameters in order to account for Coulomb collisions in a more physical way and also to treat spatially and thermally inhomogeneous systems.

In each cell, we define the species-averaged plasma coupling parameter

$$
\bar{\Gamma}_{i}=\frac{E_{C, i}}{k_{B} T_{i}},
$$


where $E_{C, i}=\left\langle q^{2}\right\rangle_{i} / 4 \pi \varepsilon_{0} \xi_{i}$ is the mean Coulomb energy per particle, $\left\langle q^{2}\right\rangle_{i}$ the particles average (squared) charge in cell $i$, and $\xi_{i}$ is a typical inter-particle distance depending on the local particle number density $n_{i}$; finally, the cell temperature $T_{i}$ is assumed to be proportional to the average kinetic energy of the particles inside the cell as $k_{B} T_{i}=\left(1 / N_{i}\right) \sum m_{j} v_{j}$. Before the collision step, the code evaluates for each cell the (multi-particle) collision probability as

$$
\mathcal{P}_{i}=\frac{1}{1+\bar{\Gamma}_{i}^{-2}}
$$

After sampling a random number $\mathcal{P}_{i}^{*}$ from a uniform distribution in the interval [0,1], the multi-particle collision occurs if $\mathcal{P}_{i}^{*} / \mathcal{P}_{i} \leq 1$.

\section{2 | Preliminary 1D kinetic simulations}

Here, we present numerical simulations of 1D systems modelling the plasma dynamics along a field line between a hot thermal bath (upstream region) and the colder wall region. In this preliminary work, we always assume regimes of strong correlation between ion and electron motion as well as fulfilment of the quasineutrality condition. In such conditions, the main contribution to the heat flux is due to electrons (see panels (b) of Figures 3 and 4); we therefore consider a single-component system representing the electrons and treat the ions as a non-interacting background adjusting itself as the electron density $n_{e}$ evolves in order to yield a globally null electric field. With such assumptions, Equation 9 becomes a standard 1D Fokker-Planck equation of the form $\partial_{t} f+v \partial_{r} f=\partial_{v}\left(v \partial_{v} f\right)$.

In our PIC-MPC code, the interaction with the hot source and the wall is modelled with standard Maxwellian thermal baths. In practice, when a simulation particle enters the hot region, its velocity $v$ is substituted with a new velocity $v^{\prime}$ taken from a Maxwellian distribution at temperature $T_{\mathrm{Hot}}$. When the particle instead hits the cold wall, it is either reflected elastically or re-immitted in the simulation domain with a velocity taken from a Maxwellian distribution at temperature $T_{\text {Cold }}$, with probabilities of one-half. Note that, with such choice, the total particle number $N_{p}$ is conserved as no particle leaves the system. In principle, it is also possible to account for particle evaporation by considering an additional velocity-dependent exclusion protocol that selects hotter particles and removes them from the system. A "stochastic evaporation" algorithm is currently under testing and will be discussed in a forthcoming publication.

Particle propagation is carried out with a standard second-order leap frog scheme, while collisions are accounted for as described in Section 3.1. All simulations discussed here were performed with fixed timestep $\delta \tau=0.01 \Omega_{P}^{-1}$, where $\Omega_{P}=$ $\sqrt{n_{e} e^{2} / m_{e} \varepsilon_{0}}$ is the plasma frequency of the system neglecting the thermal motion and extended up to $\tau=10^{3} / \Omega_{P}$.

In the kinetic simulations, we have taken the same combinations of temperature, density, and parallel length as in the two cases discussed in Section 2, yielding the two values of the collisionality $v^{\star}=60,4$. We have assumed equilibrium initial conditions by placing the particles representing the electron component homogeneously on the simulation domain $\left[0, L_{\| \mid}\right]$(i.e., constant initial electron number density $n_{e, 0}$ ), with velocities taken from a thermal distribution at temperature $T_{e, 0}$. After a short transient of about $10 \delta \tau$, the thermal baths at $T_{\text {hot }}=130 \mathrm{eV}$ and $T_{\text {cold }}=78$ and $T_{\text {hot }}=345 \mathrm{eV}$ and $T_{\text {cold }}=285 \mathrm{eV}$ for the $\nu^{\star}=60$ and $\nu^{\star}=4$ cases are applied for both cases in $x=L_{||} / 2$ and $x=0, L_{||}$, respectively.

From the initial values of the electron temperature and density $T_{e, 0}$ and $n_{e, 0}$, respectively, we derive the initial global plasma-coupling parameter $\Gamma=E_{C, 0} / k_{B} T_{0}$ that gives another measure of the strength of the system's collisionality (at least) in its initial state (i.e., at fixed $L_{||}$or at fixed $\Omega_{P}$, larger $\Gamma$ implies higher collisionality). With the present combination of parameters, we obtain $\Gamma \approx 6$ for $\nu^{\star}=60$ and $\Gamma \approx 0.66$ for $\nu^{\star}=4$.

Figure 5 shows the asymptotic equilibrium state of the two models with collisionality $v^{\star}=60$ and $4(\Gamma=6$ and 0.66$)$, in contact with a hot source at $x=L_{\| \mid} / 2$ and a cold wall at $x=0$ and $L_{\|}$. In both cases, the systems (consistently) show non-uniform density profiles with a density accumulation in correspondence with the cold source. Vice versa, the density is depleted approaching the hot source due to the larger mean velocities of particles in this region. Different from Figures 3 and 4, the electron temperature $T_{e}$ is given in units of the system's mean final temperature $T_{M}$, so that the two curves can be more easily compared being on the same scale. The initially more collisional system (i.e., $\Gamma=6$ ) has a quasi-linear temperature profile over a broader interval of the parallel coordinate $x$ (i.e., $0.5 \leq x / L_{||} \leq 0.73$ ), while the weakly collisional system has a more complex asymptotic temperature profile characterized by several slope changes and a flat central region (remarkably similar to the corresponding curve in Figure 4, panel a), pointing to a highly non-local heat transport regime. Remarkably, in both cases, the final electron pressure $P_{e} \propto n_{e} T_{e}$ is spatially constant as we clearly observe $n_{e} \propto T_{e}^{-1}$ at $\tau=10^{3}$ (panel c).

For the same systems of Figure 5, Figure 6 shows the initial and final velocity distributions $f(v)$ (panels a, b) and the sections of (half of) the numerically recovered phase-space distribution function $f(x, v)$ at $x=L_{||} / 2$ and $x=L_{||}$(panel c). The strongly interacting model with $v^{\star}=60$ presents a final $f(v)$ that is well described by a Gaussian, while the model with $v^{\star}=4$ has a clearly non-thermal final velocity distribution. Both cases, however, appear to be colder in their final state with respect to their initial states. For what concerns the phase-space distribution, while in both cases $f(x, v)$ clearly approaches a thermal distribution in 

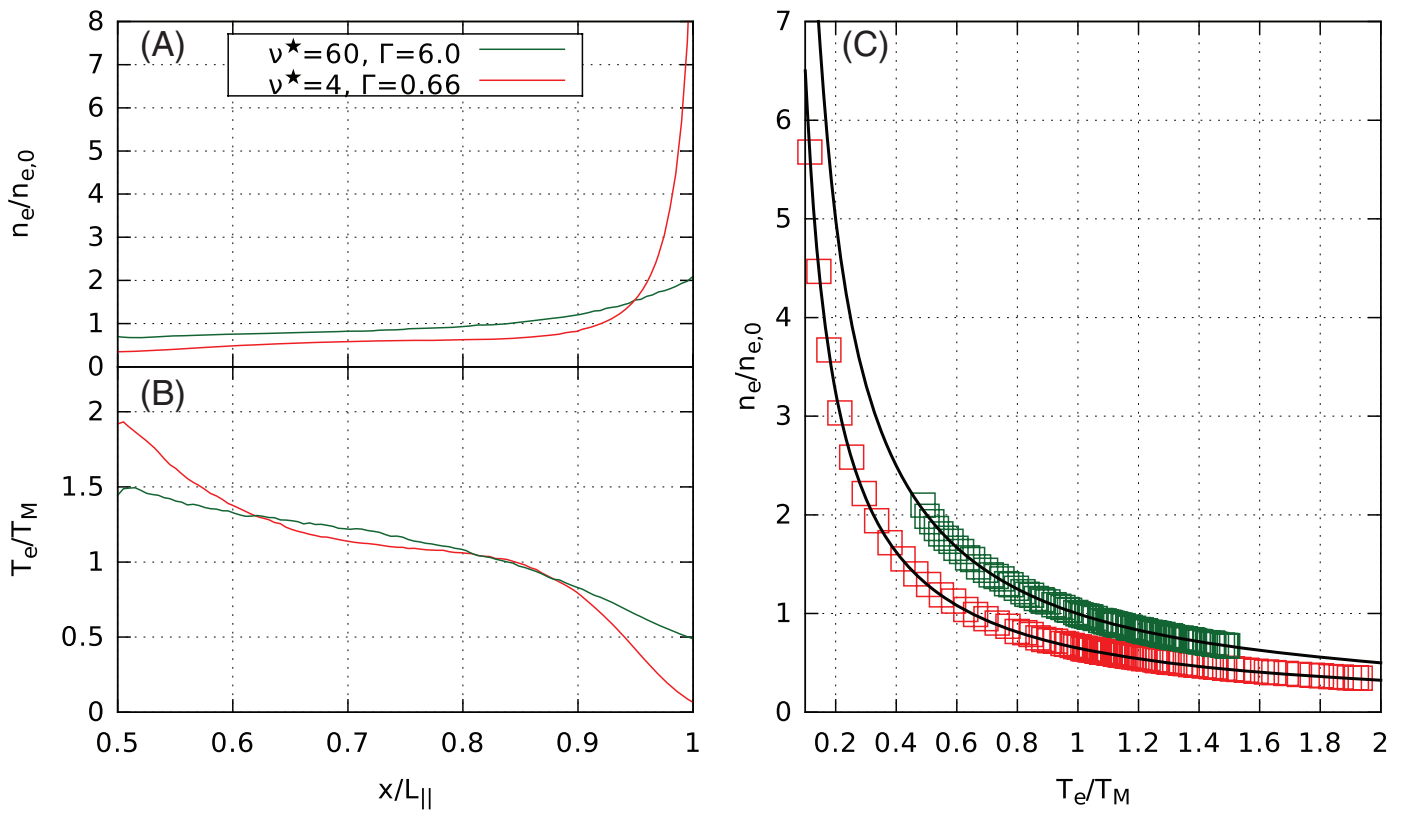

FIGURE 5 For two models with $\Gamma=6$ and 0.66 : final electron number density $n_{e}$ (a) and temperature profile $T_{e}$ (b) as function of the parallel coordinate $x / L_{\|}$, and density temperature relation (squares) and best-fit curves (solid lines), (c). Parallel density and temperature profiles are given only for $L_{\|} / 2 \leq x \leq L_{\|}$ as they are perfectly symmetrical in the other half of the simulation domain
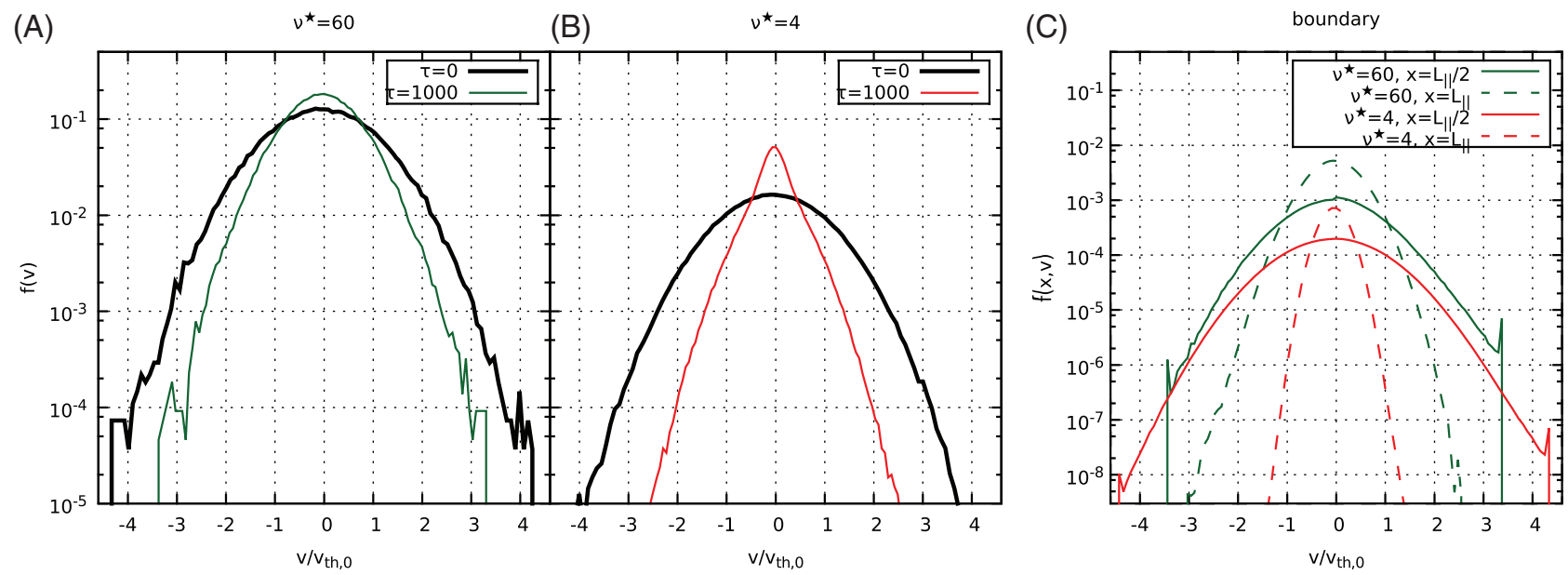

FIGURE 6 Velocity distributions $f(v)$ for the case with $v^{\star}=60$ (a) and $v^{\star}=4$ (b). The thin solid lines correspond to the equilibrium state (reached at around $\tau \approx 10^{3}$ ), while the heavy solid line mark the initial velocity distributions. Sections of the phase-space distribution function $f(x, v)$ at the cold wall (dashed lines) and hot (solid lines lines) (c). In all cases, the velocities are normalized with respect to the initial thermal velocity $v_{t h, 0}$

correspondence with the cold point, the structure of $f(x, v)$ at $x=L_{||} / 2$ is somewhat more complicated and characterized by a fatter tail at positive velocities (i.e., corresponding to particles moving towards the cold point). In addition, in correspondence with the highest velocities attained by the particles, two peak-like structures can be clearly seen. We interpret this feature as a finite-size effect due to the almost vanishing lifetime of larger velocities reaching the cold wall. In fact, at fixed $\nu^{\star}, \Gamma$, and thermal baths temperatures $T_{\text {hot }}$ and $T_{\text {cold }}$, such peaks tend to disappear for increasing $L_{||}$.

\section{4 | CONCLUSION AND OUTLOOK}

We have shown the impact of FL techniques on the computation of heat flux on divertor tokamak simulations. We have proposed the implementation of a non-local approach in a 1D fluid model, and we have presented the numerical results obtained with SOLEDGE1D at medium and high collisionality. In the second part of the paper, PIC-MPC kinetic simulations are presented. They offer a particle-based approach that appears to be more suitable to study transient regimes and relaxation processes. Remarkably, for the case studies discussed in this paper, we found good agreement between this approach and the fluid 
modelling, suggesting that further evolutions of the fluid scheme could be tested against more detailed PIC-MPC simulations, including more species and the effect of the self-consistent fields.

\section{ACKNOWLEDGMENTS}

This work has been carried out within the framework of the EUROfusion Consortium and has received funding from the Euratom research and training programme 2014-2018 under grant agreement No 633053 for the project WP17-ENR-CEA-01. The views and opinions expressed herein do not necessarily reflect those of the European Commission. This work was granted access to the HPC resources of Aix-Marseille University financed by the project Equip@ Meso (ANR-10-EQPX-29-01) of the programme "Investissments d'Avenir" supervised by the Agence Nationale pour la Recherche. P.F.D.C. acknowledges partial support by the INFN project DYNSYSMATH 2017.

\section{NOTES}

* Note that particles are to be thought as a discrete sampling of $f$, rather than actual "particles".

$\dagger$ In our implementation, the mesh used in the MPC step is the same as the one used by the PIC code to compute electromagnetic fields.

\section{REFERENCES}

[1] H. Bufferand, G. Ciraolo, Y. Marandet, J. Bucalossi, P. Ghendrih, J. Gunn, N. Mellet, P. Tamain, R. Leybros, N. Fedorczak, F. Schwander, E. Serre, Nucl. Fusion 2015, 55, 053025 .

[2] S. Wiesen, D. Reiter, V. Kotov, M. Baelmans, W. Dekeyser, A. S. Kukushkin, S. W. Lisgo, R. A. Pitts, V. Rozhansky, G. Saibene, I. Veselova, S. Voskoboynikov, J. Nucl. Mater. 2015, 463, 480 .

[3] C. Guillemaut, R. A. Pitts, A. S. Kukushkin, J. P. Gunn, J. Bucalossi, G. Arnoux, P. Belo, S. Brezinsek, M. Brix, G. Corrigan, S. Devaux, J. Flanagan, M. Groth, D. Harting, A. Huber, S. Jachmich, U. Kruezi, M. Lehnen, C. Marchetto, S. Marsen, A. G. Meigs, O. Meyer, M. Stamp, J. D. Strachan, S. Wiesen, M. Wischmeier and JET EFDA Contributors, Nucl. Fusion 2014, 54, 093012.

[4] K. Shimizu, T. Takizuka, K. Ohya, K. Inai, T. Nakano, A. Takayama, H. Kawashima, K. Hoshino, Nucl. Fusion 2009, 49 , 065028.

[5] T. D. Rognlien, P. N. Brown, R. B. Campbell, T. B. Kaiser, D. A. Knoll, P. R. McHugh, G. D. Porter, M. E. Rensink, G. R. Smith, Contrib. Plasma Phys. 1994, 34, 362.

[6] L. Spitzer, R. Härm, Phys. Rev. 1953, 89, 977. https://doi.org/10.1103/PhysRev.89.977.

[7] P. Stangeby, The Plasma Boundary of Magnetic Fusion Devices, Institute of Physics Publishing, Bristol 2000.

[8] W. Fundamenski, Plasma Phys. Controlled Fusion 2005, 47, R163.

[9] J. Bucalossi, M. Missirlian, P. Moreau, F. Samaille, E. Tsitrone, D. van Houtte, T. Batal, C. Bourdelle, M. Chantant, Y. Corre, X. Courtois, L. Delpech, L. Doceul, D. Douai, H. Dougnac, F. Faïsse, C. Fenzi, F. Ferlay, M. Firdaouss, L. Gargiulo, P. Garin, C. Gil, A. Grosman, D. Guilhem, J. Gunn, C. Hernandez, D. Keller, S. Larroque, F. Leroux, M. Lipa, P. Lotte, A. Martinez, O. Meyer, F. Micolon, P. Mollard, E. Nardon, R. Nouailletas, A. Pilia, M. Richou, S. Salasca, J.-M. Travère, Fusion Eng. Des. 2014, 89, 907.

[10] A. V. Chankin, D. P. Coster, R. Dux, Ch. Fuchs, G. Haas, A. Herrmann, L. D. Horton, A. Kallenbach, M. Kaufmann, Ch. Konz, K. Lackner, C. Maggi, H. W. Müller, J. Neuhauser, R. Pugno, M. Reich, W. Schneider, Plasma Phys. Controlled Fusion 2006, $48,839$.

[11] G. Ciraolo, H. Bufferand, J. Bucalossi, Ph. Ghendrih, P. Tamain, Y. Marandet, M. Valentinuzzi, J. Denis, N. Fedorczak, E. Hodille, N. Mellet, B. Pegourie, C. Grisolia, C. Bourdelle, E. Tsitrone, D. Galassi, R. Leybros, G. Giorgiani, E. Serre, Nucl. Mater. Energy 2017, $12,187$.

[12] J. F. Luciani, P. Mora, J. Virmont, Phys. Rev. Lett. 1983, 51, 1665.

[13] H. Bufferand, G. Ciraolo, P. Di Cintio, N. Fedorczak, Ph. Ghendrih, S. Lepri, R. Livi, Y. Marandet, E. Serre, P. Tamain, Contrib. Plasma Phys. 2018, https://doi. org/10.1002/ctpp.201700162.

[14] D. Tskhakaya, F. Subba, X. Bonnin, D. P. Coster, W. Fundamenski, R. A. Pitts, JET EFDA Contributors, Contrib. Plasma Phys. 2008, 48, 89. https://doi.org/10. 1002/ctpp.200810015.

[15] A. Froese, T. Takizuka, M. Yagi, Contrib. Plasma Phys. 2012, 52, 534. https://doi.org/10.1002/ctpp.201210045.

[16] K. Dressler, Math. Methods Appl. Sci. 1990, 12, 471.

[17] E. J. Allen, H. D. Victory, Phys. A Stat. Mech. Appl. 1994, 209, 318.

[18] A. R. Bell, A. P. L. Robinson, M. Sherlock, R. J. Kingham, W. Rozmus, Plasma Phys. Controlled Fusion 2006, 48, R37. https://doi.org/10.1088/0741-3335/48/3/ R01.

[19] M. N. Rosenbluth, W. M. MacDonald, D. L. Judd, Phys. Rev. 1957, 107, 1. https://doi.org/10.1103/PhysRev.107.1.

[20] W. M. MacDonald, M. N. Rosenbluth, W. Chuck, Phys. Rev. 1957, 107, 350. https://doi.org/10.1103/PhysRev.107.350.

[21] R. W. Hockney, J. W. Eastwood, Computer Simulation Using Particles, New York, McGraw-Hill 1981.

[22] A. Malevanets, R. Kapral, J. Comput. Phys. 1999, 110, 8605.

[23] P. Di Cintio, R. Livi, S. Lepri, G. Ciraolo, Phys. Rev. E 2017, 95, 043203.

[24] P. Di Cintio, R. Livi, H. Bufferand, G. Ciraolo, S. Lepri, M. J. Straka, Phys. Rev. E 2015, 92, 062108.

[25] H. Bufferand, G. Ciraolo, P. Ghendrih, P. Tamain, F. Bagnoli, S. Lepri, R. Livi, J. Phys.: Conf. Ser. $2010,260,012005$.

[26] H. Bufferand, G. Ciraolo, P. Ghendrih, S. Lepri, R. Livi, Phys. Rev. E 2013, 87, 023102. 